\title{
Healthy eating index in a nationally representative sample of children and adolescents by socio-demographic characteristics: the Weight disorders survey of the CASPIAN-IV Study
}

\author{
Golgis Karimi ${ }^{1 \oplus}$, Motahar Heidari-Beni ${ }^{2 \oplus}$, Roya Riahi ${ }^{2 \oplus}$, Mostafa Qorbani ${ }^{3,4 \oplus,}$ \\ Roya Kelishadi ${ }^{2}$
}

${ }^{1}$ Cellular and Molecular Biology Research Center, Shahid Beheshti University of Medical Sciences, Tehran, Iran; ${ }^{2}$ Department of Pediatrics, Child Growth and Development Research Center, Research Institute for Primordial Prevention of Non-Communicable Disease, Isfahan University of Medical Sciences, Isfahan, Iran; ${ }^{3}$ Non-Communicable Diseases Research Center, Alborz University of Medical Sciences, Karaj, Iran; ${ }^{4}$ Chronic Diseases Research Center, Endocrinology and Metabolism Population Sciences Institute, Tehran University of Medical Sciences, Tehran, Iran.

\begin{abstract}
Background. To date, the diet quality of Iranian students in relation to socio-demographic characteristics was not studied. The present study aimed to explore the association between the healthy eating index and sociodemographic characteristics among a nationally representative sample of Iranian children and adolescents.

Methods. This nationwide study was conducted in 5187 children and adolescents, aged 6-18 years. Data regarding socio-demographic variables, lifestyle factors, family and student dietary habits, and quality of life were gathered via validated questionnaires. The Alternate Healthy Eating Index-2010 (AHEI-2010) was used to calculate diet quality scores.

Results. The odds of high diet quality was $24 \%$ lower in adolescents (13-18years) compared to children aged 6-12 years (OR 0.76, CI 0.64-0.89, $\mathrm{p}=0.001$ ). Students in families with moderate (OR 1.30, CI 1.13-1.49, $\mathrm{p}<0.001$ ) and high socioeconomic status (OR 1.36, CI 1.18-1.57, $\mathrm{p}<0.001$ ) were $30 \%$ and $36 \%$ more likely to have a higher diet quality score, respectively. Lower mean AHEI-2010 scores (CI) were found for low socio-economic status (46.18-47.10), adolescents 47.40 (46.94-47.82), boys 47.51 (47.14-47.88) and South-East area 47.19 (46.54-49.15) $(\mathrm{p}<0.05)$ due to lower intake of fruits and vegetables and high intake of sodium and sugar-sweetened beverages.
\end{abstract}

Conclusions. The overall diet quality of Iranian children and adolescents was low with disparities across sociodemographic variables notably age and familial socio-economic status.

Key words: diet quality, socio-demographic, children, adolescents.

In recent years, there has been a change in food habits and dietary patterns of people from healthy to unhealthy and low nutritive content foods. These changes vary from one region to another ${ }^{1}$ and may be responsible for the increased prevalence of cardio-metabolic disorders like overweight and obesity ${ }^{2}$

$凶$ motahar.heidari@nutr.mui.ac.ir

Motahar Heidari-Beni

Received 1st October 2019, revised 1st December 2019, 1st February 2020, accepted 16th February 2020. especially in children and adolescents.-5 Some earlier studies suggest that early life nutrition significantly contributes to childhood overweight or obesity ${ }^{6,7}$ which may track to adulthood resulting in lifelong obesity and its comorbidities battle like cardiovascular disease (CVD). ${ }^{8}$ Healthy and proper nutrition is an essential factor for children's health and growth as well as their quality of life that tends to track into adulthood. ${ }^{1}$ Findings showed that dietary patterns including a variety of fruits, vegetables, whole grains, low-fat dairy products, and lean 
meats reduce the risk of non-communicable diseases (NCDs) such as type 2 diabetes, some cancers, CVD, and osteoporosis., ${ }^{9} 10$ According to the earlier investigations, the incidence of the aforementioned disease was higher in populations with low socioeconomic status. ${ }^{1,2}$ Therefore there is a need to understand the dietary patterns of a population and provide a report of their nutritional status on a timely basis in order to identify sub-populations requiring intervention as well as getting an insight into how dietary patterns and diet quality may contribute to NCDs., ${ }^{3,4}$ Diet quality which assesses quality and variety of the diet and shows the association between whole foods and health status, rather than just nutrients, is an effective tool to elucidate the relation between nutritional status and health. ${ }^{5,6}$ The healthy eating index (HEI) is a measure of diet quality that can be used to evaluate nutrition interventions and education programs. ${ }^{16}$ It has been reported that diet quality, as well as food choices, are influenced by a number of factors including socioeconomic, individual and environmental effects. ${ }^{17,18}$ Although a number of recent studies demonstrate a positive association between socioeconomic status and indicators of diet quality, ${ }^{19,20}$ few studies have assessed the association between sociodemographic and consumption patterns of some food groups. ${ }^{4,7}, 8$ To the best of our knowledge no study has assessed such association among Iranian children and adolescents. Therefore, we evaluated the association between healthy eating index as a diet quality indices and socio-demographic factors in a nationally representative sample of Iranian children and adolescents

\section{Material and Methods}

\section{Study population}

This nationwide cross-sectional study was conducted in the framework of the Childhood and Adolescence Surveillance and Prevention of Adult Non-communicable Disease" (CASPIAN-IV) study which included a large group of Iranian children and adolescents aged 6-18 years old, living in urban and rural areas of 31 provinces in Iran. ${ }^{23}$ Participants were divided into two groups of age including children (6-12years old) and adolescents (13-18 years old). Detailed information about the study design, participants and data collection method has been published previously. ${ }^{24}$

Study protocols were reviewed and approved by the ethical committees of Isfahan University of Medical Sciences (189130/2011-2012). After a complete explanation of the study objectives and protocols, written informed consent was obtained from the parents and students.

All students were asked to fill in validated questionnaires and a group of health expertise supervised them. Data for socio-demographic, perinatal factors, lifestyle factors, as well as student's and familial history of chronic disease were collected.

\section{Dietary intake assessment}

A validated 168-items semi-quantitative food frequency questionnaire (sq-FFQ) was administered by participants to assess their dietary intake. The FFQ consisted of a list of foods with a standard serving size commonly consumed by Iranians. All students were asked for the amount and frequency of different food items during the last year based on daily, weekly, or monthly intake. The reported frequency for each food item was then converted to daily intake. ${ }^{25}$ All dietary information was entered into the Iranian Food Consumption Program (IFCP), designed by Isfahan Cardiovascular Research Center (ICRC) and analyzed. ${ }^{9}$ IFCP was designed based on the Iranian food composition table. ${ }^{10}$ Energy and nutrient intakes were estimated using IFCP.

\section{Anthropometric assessment}

Weight and height were measured by a trained person under standard protocol as follows; weight was measured in minimal clothes and barefoot nearest to the $200 \mathrm{~g}$, height was measured without shoes to the nearest 0.1 
$\mathrm{cm}$. Body mass index (BMI) was calculated by dividing weight $(\mathrm{kg})$ to height squared $\left(\mathrm{m}^{2}\right)$. Waist circumference (WC) was measured between the iliac crest and the lowest rib to the nearest $0.2 \mathrm{~cm}$.

\section{Socioeconomic status (SES) assessment}

To determine the SES of participants, the previously approved methodology "Progress in the International Reading Literacy Study (PIRLS)" for Iran was used. Using principal component analysis (PCA), parents' education, parents' job, possessing a private car, school type (public/private), type of home (private/ rented), and having a personal computer variable were summarized under one main component, categorized into four quartiles. Through an ascending grade, the first quartile was defined as the "lowest SES" and the fourth quartile as the "highest SES" groups. ${ }^{11}$

\section{Calculating HEI scores}

According to the recent studies, Alternate Healthy Eating Index-2010 (AHEI-2010) in comparison to Healthy Eating Index-2010 (HEI-2010) has a stronger link to chronic diseases and should thus forecast risks better. ${ }^{12}$ Therefore, in the present study AHEI-2010 was used to calculate the scores. The AHEI2010 is based on 11 components; for six components, the highest intake considered as desired including vegetables, fruits, whole grains, nuts, legumes, long-chain omega-3 fats (docosahexaenoic acid and eicosapentaenoic acid), and polyunsaturated fatty acids (PUFAs). For one component (alcohol) moderate intakes are supposed to be ideal while for the rest of the components lower intake posit to be desired including sodium, sugar-sweetened beverages (SSB), red and processed meat, and trans fats. Each component is given a score between 0 to 10. Total scores range from $0-110$ by summing up the score of each component. A higher score represents a better diet quality. ${ }^{26}$ In other words, AHEI-2010 makes no explicit division between adequacy and moderation. ${ }^{12}$

\section{Statistical analysis}

Continuous variables are showed as mean $(95 \% \mathrm{CI})$ and categorical variables as a percent (95\% CI). Study variables across socioeconomic status and socioeconomic status of the living area were assessed using ANOVA test and across gender and age categories were assessed by t-test. Considering the hierarchical structure of our data, a multilevel ordered logistic regression model (with two-level) was used to assess the association between quartile of the healthy eating index and sociodemographic characteristics, taking account the effect of a mix of individual-level (first level) and provincial level (second level) factors. Multilevel modeling adequately illustrates the unexplained variability of the nested structure, which is often hard to explain in the single-level approach. ${ }^{27}$ The results of multilevel models were presented as odds ratios (OR) with 95\% CI. A P-value of less than 0.05 was considered statistically significant in all analyses. All statistical analyses were conducted by Stata 11.2 (StataCorp LP, College Station, TX)

\section{Results}

A total of 5187 students were analyzed, $52.6 \%$ were boys and $72.4 \%$ lived in the urban area. Table I represents the socio-demographic characteristics of participants. Overall, 34\% of children and adolescents lived in provinces with the highest socioeconomic status (central), and $15.6 \%$ of those lived in the southeast area with the lowest socioeconomic status.

Body composition and dietary intake of participants according to family socioeconomic status, socioeconomic status of living area, gender and age are presented in Tables II and III, respectively. Mean body weight, waist circumferences, and body mass index was significantly higher in students with high family socioeconomic status and those living in the central region (with the highest SES) than others ( $p$-value $<0.05$ ). 
Table I. Demographic characteristics of participants.

\begin{tabular}{llll}
\hline & & Percent & 95\%CI \\
\hline \multirow{2}{*}{ Age } & 6-12 years & $61.7 \%$ & $(60.463 .3)$ \\
& 13-18 years & $38.3 \%$ & $(36.639 .6)$ \\
\multirow{5}{*}{ Living area } & Boys & $52.6 \%$ & $(50.853 .8)$ \\
& Girls & $47.4 \%$ & $(46.149 .2)$ \\
& Urban & $72.4 \%$ & $(69.872 .6)$ \\
Socio-economic status of living region & Rural & $27.5 \%$ & $(27.430 .2)$ \\
& Lowest (Southeast) & $15.9 \%$ & $(17.119 .4)$ \\
& Second Low (North-Northeast) & $19.5 \%$ & $(21.824 .3)$ \\
& Second High SES (West) & $30.6 \%$ & $(32.435 .3)$ \\
Family socio-economic status & Highest SES (Central) & $34 \%$ & $(23.626 .2)$ \\
& Low & $33.2 \%$ & $(31.834 .6)$ \\
& Moderate & $33.4 \%$ & $(32.0 .34 .8)$ \\
& High & $33.4 \%$ & $(32.034 .8)$ \\
\hline
\end{tabular}

Children and adolescents with high SES and those who lived in the central (with the highest SES) had significantly higher intakes of total calorie, carbohydrate, protein, fruit, vegetables, processed meat, and lower intake of total fat and PUFAs than others ( $p$-value $<0.05$ ) (Tables II and III).

The mean healthy eating index (HEI) in participants who lived in the southeast (with the lowest SES) and those with low family SES was significantly lower than other students (p-value < 0.05) (Tables II and III)

Consumption of total fat, sodium, and vegetables was significantly higher among girls than boys ( $p$-value $<0.05$ ), while boys had a higher intake of SSB, nuts and legumes (Table IV).

Adolescents (students aged 13-18 years) consumed a significantly higher amount of sodium, whole grain, and SSB than children (students aged 6-12 years) ( $p$-value $<0.05)$. The mean score $( \pm$ SD) of HEI in boys $(47.51 \pm 9.22)$ was lower than girls $(48.39 \pm 8.99)$. Adolescents $(47.40 \pm 8.85)$ had lower HEI score compared to the children $(48.25 \pm 9.52)$ (p-value $<0.05)$ (Table IV).

Association between socio-demographic characteristics and HEI (at the provincial level) using multilevel ordinal logistic regression is shown in Table V. At an individual level, odds of higher HEI score in students aged 13-18 years, was $24 \%$ lower than students aged 6-12 years, (OR $0.76<95 \% \mathrm{CI}$ : 0.64 to $0.89, \mathrm{P}<0.05$ ). Students with high (OR $1.36<95 \%$ CI: 1.18 to 1.57, P < 0.05) and moderate (OR $1.30<95 \%$ CI: 1.13 to $1.49, \mathrm{P}<0.05)$ family SES were $36 \%$ and $30 \%$ more likely to have higher HEI score than students with low family SES, respectively. SES of living area at the provincial level was not significantly associated with HEI scores ( $p$-value $>0.05$ ). Total diet quality scores based on socio-demographic variables categories are summarized in Figure 1. Based on this figure girls with high SES and living in north/northeast of Iran had better diet quality.

\section{Discussion}

The overall diet quality of Iranian children and adolescents based on a mean HEI score was less than half the maximum score. Such a score is due to the high consumption of sodium, SSB, processed meat, and a lower intake of whole grains, fruits, vegetables, nuts, and legumes. The results of the present study indicate an association between diet quality and sociodemographic characteristics. A higher score of diet quality was significantly associated with 
Table II. Body composition and dietary intakes of children according to family socio-economic status.

\begin{tabular}{|c|c|c|c|c|}
\hline \multirow{2}{*}{ Mean $(95 \% \mathrm{CI})$} & \multicolumn{3}{|c|}{ Socio-economic status } & \multirow{2}{*}{$P$ for trend } \\
\hline & Low & Moderate & High & \\
\hline Body weight (kg) & $\begin{array}{c}38.48 \\
(37.739 .25)\end{array}$ & $\begin{array}{c}41.09 \\
(40.2241 .96)\end{array}$ & $\begin{array}{c}46.47 \\
(45.5447 .4)\end{array}$ & $<0.001^{*}$ \\
\hline Waist circumference $(\mathrm{cm})$ & $\begin{array}{c}63.8 \\
(63.2764 .42)\end{array}$ & $\begin{array}{c}66 \\
(65.4366 .67)\end{array}$ & $\begin{array}{c}69.6 \\
(68.9570 .29)\end{array}$ & $<0.001^{*}$ \\
\hline BMI $\left(\mathrm{kg} / \mathrm{m}^{2}\right)$ & $\begin{array}{c}18 \\
(17.81 \text { 18.28) }\end{array}$ & $\begin{array}{c}18.9 \\
(18.6019 .15)\end{array}$ & $\begin{array}{c}19.9 \\
(19.6420 .16)\end{array}$ & $<0.001^{*}$ \\
\hline Total Energy intake (Kcal/day) & $\begin{array}{c}2445.1 \\
(2401.62488 .5)\end{array}$ & $\begin{array}{c}2527.3 \\
(2487.72566 .8)\end{array}$ & $\begin{array}{c}2598.4 \\
(2558.52638 .2)\end{array}$ & $<0.001^{*}$ \\
\hline Carbohydrate (\% Kcal) & $\begin{array}{c}54.9 \\
(54.555 .36)\end{array}$ & $\begin{array}{c}55.6 \\
(55.2055 .96)\end{array}$ & $\begin{array}{c}55.6 \\
(55.2255 .96)\end{array}$ & $0.022^{*}$ \\
\hline Fat $(\% \mathrm{Kcal})$ & $\begin{array}{c}34.3 \\
(33.9134 .69)\end{array}$ & $\begin{array}{c}33.3 \\
(32.9433 .62)\end{array}$ & $\begin{array}{c}32.8 \\
(32.4833 .13)\end{array}$ & $<0.001^{*}$ \\
\hline Protein (\% Kcal) & $\begin{array}{c}12.7 \\
(12.5312 .83)\end{array}$ & $\begin{array}{c}13.4 \\
(13.2813 .54)\end{array}$ & $\begin{array}{c}14.01 \\
(13.8714 .15)\end{array}$ & $<0.001^{*}$ \\
\hline Fiber (gr/day) & $\begin{array}{c}26.93 \\
(26.2027 .65)\end{array}$ & $\begin{array}{c}28.51 \\
(27.8529 .18)\end{array}$ & $\begin{array}{c}29.51 \\
(28.8430 .19)\end{array}$ & $<0.001^{*}$ \\
\hline Sodium (mg/day) & $\begin{array}{c}6150.40 \\
(5720.26580 .6)\end{array}$ & $\begin{array}{c}6053.35 \\
(5453.26653 .5)\end{array}$ & $\begin{array}{c}6033.02 \\
(5442.16623 .9)\end{array}$ & 0.77 \\
\hline Long chain omega 3 fatty acid & $\begin{array}{c}0.20 \\
(0.180 .23)\end{array}$ & $\begin{array}{c}0.21 \\
(0.180 .23)\end{array}$ & $\begin{array}{c}0.22 \\
(0.200 .24)\end{array}$ & 0.17 \\
\hline Poly unsaturated fatty acid (g/day) & $\begin{array}{c}18.74 \\
(18.2519 .23)\end{array}$ & $\begin{array}{c}17.76 \\
(17.3418 .18)\end{array}$ & $\begin{array}{c}17.46 \\
(17.0417 .88)\end{array}$ & $<0.001^{*}$ \\
\hline Fruits (gr/day) & $\begin{array}{c}226.68 \\
(214.84238 .51)\end{array}$ & $\begin{array}{c}313.59 \\
(298.70328 .49)\end{array}$ & $\begin{array}{c}380.58 \\
(363.88397 .28)\end{array}$ & $<0.001^{*}$ \\
\hline Vegetables (gr/day) & $\begin{array}{c}296.41 \\
(286.70306 .12)\end{array}$ & $\begin{array}{c}329.88 \\
(319.70340 .06)\end{array}$ & $\begin{array}{c}340.84 \\
(330.28351 .39)\end{array}$ & $<0.001^{*}$ \\
\hline Whole grain (gr/day) & $\begin{array}{c}40.33 \\
(37.4343 .23)\end{array}$ & $\begin{array}{c}44.14 \\
(41.0547 .24)\end{array}$ & $\begin{array}{c}41.61 \\
(38.8644 .36)\end{array}$ & 0.54 \\
\hline Nuts \& legumes (gr/day) & $\begin{array}{c}49.81 \\
(47.4652 .16)\end{array}$ & $\begin{array}{c}51.08 \\
(48.9253 .25)\end{array}$ & $\begin{array}{c}50.15 \\
(47.9652 .33)\end{array}$ & 0.84 \\
\hline Sugar-sweetened beverage (gr/day) & $\begin{array}{c}77.81 \\
(72.4483 .18)\end{array}$ & $\begin{array}{c}85.73 \\
(79.9491 .53)\end{array}$ & $\begin{array}{c}85.28 \\
(79.9590 .61)\end{array}$ & 0.06 \\
\hline Processed meat (gr/day) & $\begin{array}{c}25.20 \\
(23.7826 .62)\end{array}$ & $\begin{array}{c}32.75 \\
(30.9734 .52)\end{array}$ & $\begin{array}{c}39.55 \\
(37.4741 .63)\end{array}$ & $<0.001^{*}$ \\
\hline Healthy eating index (HEI) & $\begin{array}{c}46.63 \\
(46.1847 .10)\end{array}$ & $\begin{array}{c}48.53 \\
(48.0649 .00)\end{array}$ & $\begin{array}{c}48.67 \\
(48.1949 .15)\end{array}$ & $<0.001^{*}$ \\
\hline
\end{tabular}

${ }^{*}$ p-value $<0.05$ considered as statistically significant

family SES while such an association was not observed for gender and socioeconomic status of living region. Children with high SES showed higher body weight, waist circumference,
BMI, energy intake, protein, fiber, fruits, and vegetables intake coupled with lower fat and PUFAs intake. Furthermore, as age increased diet quality has decreased. In fact, a significant 
Table III. Body composition and dietary intakes of children according to socio-economic status of living region.

\begin{tabular}{|c|c|c|c|c|c|}
\hline \multirow[b]{2}{*}{ Mean (95\%CI) } & \multicolumn{4}{|c|}{ Socio-economic status of living area } & \multirow[b]{2}{*}{$P$ for trend } \\
\hline & $\begin{array}{l}\text { Lowest } \\
\text { (Southeast) }\end{array}$ & $\begin{array}{l}\text { Second Low } \\
\text { (North/ } \\
\text { Northeast) }\end{array}$ & $\begin{array}{l}\text { Second High SES } \\
\text { (West) }\end{array}$ & $\begin{array}{l}\text { Highest SES } \\
\text { (Central) }\end{array}$ & \\
\hline Body weight (kg) & $\begin{array}{c}38.49 \\
(37.3639 .62)\end{array}$ & $\begin{array}{c}44.91 \\
(43.8545 .98)\end{array}$ & $\begin{array}{c}40.62 \\
(39.8241 .42)\end{array}$ & $\begin{array}{c}44.29 \\
(43.2145 .37)\end{array}$ & $<0.001^{*}$ \\
\hline Waist circumference $(\mathrm{cm})$ & $\begin{array}{c}62.75 \\
(61.9463 .57)\end{array}$ & $\begin{array}{c}69.06 \\
(68.3069 .82)\end{array}$ & $\begin{array}{c}66.21 \\
(65.6066 .83)\end{array}$ & $\begin{array}{c}67.63 \\
(66.9168 .36)\end{array}$ & $<0.001^{*}$ \\
\hline Body mass index $\left(\mathrm{kg} / \mathrm{m}^{2}\right)$ & $\begin{array}{c}17.43 \\
(17.1517 .71)\end{array}$ & $\begin{array}{c}19.64 \\
(19.3519 .92)\end{array}$ & $\begin{array}{c}19.01 \\
(18.7319 .28)\end{array}$ & $\begin{array}{c}19.39 \\
(19.0819 .71)\end{array}$ & $<0.001^{*}$ \\
\hline $\begin{array}{l}\text { Total Energy intake } \\
\text { (Kcal/day) }\end{array}$ & $\begin{array}{c}2321.40 \\
(2265.82377)\end{array}$ & $\begin{array}{c}2531.31 \\
(2482.12580 .5)\end{array}$ & $\begin{array}{c}2659.83 \\
(2622.42697 .2)\end{array}$ & $\begin{array}{c}2579.73 \\
(2544.52614 .9)\end{array}$ & $<0.001^{*}$ \\
\hline Carbohydrate (\% Kcal) & $\begin{array}{c}52.85 \\
(52.3153 .39)\end{array}$ & $\begin{array}{c}57.14 \\
(56.7157 .56)\end{array}$ & $\begin{array}{c}55.20 \\
(54.8255 .57)\end{array}$ & $\begin{array}{c}55.91 \\
(55.5756 .25)\end{array}$ & $<0.001^{*}$ \\
\hline Fat (\% Kcal) & $\begin{array}{c}35.92 \\
(35.4036 .44)\end{array}$ & $\begin{array}{c}31.94 \\
(31.5432 .34)\end{array}$ & $\begin{array}{c}33.92 \\
(33.6034 .25)\end{array}$ & $\begin{array}{c}32.84 \\
(32.5433 .14)\end{array}$ & $<0.001^{*}$ \\
\hline Protein (\% Kcal) & $\begin{array}{c}12.98 \\
(12.7613 .20)\end{array}$ & $\begin{array}{c}13.36 \\
(13.2113 .1)\end{array}$ & $\begin{array}{c}13.14 \\
(13.0113 .27)\end{array}$ & $\begin{array}{c}13.52 \\
(13.4013 .65)\end{array}$ & $<0.001^{*}$ \\
\hline Fiber (gr/day) & $\begin{array}{c}22.02 \\
(21.3322 .71)\end{array}$ & $\begin{array}{c}28.62 \\
(27.8829 .36)\end{array}$ & $\begin{array}{c}32.40 \\
(31.6433 .16)\end{array}$ & $\begin{array}{c}29.48 \\
(28.8530 .10)\end{array}$ & $<0.001^{*}$ \\
\hline Sodium (mg/day) & $\begin{array}{c}6085.59 \\
(5737.96433 .3)\end{array}$ & $\begin{array}{c}6284.26 \\
(5680.86887 .8)\end{array}$ & $\begin{array}{c}6095.83 \\
(5680.86887 .8)\end{array}$ & $\begin{array}{c}5767.96 \\
(5292.46243 .5)\end{array}$ & 0.37 \\
\hline $\begin{array}{l}\text { Long chain omega } 3 \text { fatty } \\
\text { acid }\end{array}$ & $\begin{array}{c}0.32 \\
(0.280 .36)\end{array}$ & $\begin{array}{c}0.22 \\
(0.200 .24)\end{array}$ & $\begin{array}{c}0.18 \\
(0.160 .20)\end{array}$ & $\begin{array}{c}0.15 \\
(0.130 .16)\end{array}$ & $<0.001^{*}$ \\
\hline $\begin{array}{l}\text { Poly unsaturated fatty } \\
\text { acid (gr/day) }\end{array}$ & $\begin{array}{c}19.48 \\
(18.7720 .18)\end{array}$ & $\begin{array}{c}17.23 \\
(16.7317 .74)\end{array}$ & $\begin{array}{c}18.88 \\
(18.4819 .27)\end{array}$ & $\begin{array}{c}17.79 \\
(17.4018 .18)\end{array}$ & $0.003^{*}$ \\
\hline Fruits (gr/day) & $\begin{array}{c}178.42 \\
(167.22189 .62)\end{array}$ & $\begin{array}{c}320.26 \\
(302.60337 .93)\end{array}$ & $\begin{array}{c}295.36 \\
(281.99308 .72)\end{array}$ & $\begin{array}{c}379.30 \\
(364.93393 .67)\end{array}$ & $<0.001^{*}$ \\
\hline Vegetables (gr/day) & $\begin{array}{c}275.07 \\
(262.39287 .75)\end{array}$ & $\begin{array}{c}328.96 \\
(316.96340 .95)\end{array}$ & $\begin{array}{c}336.00 \\
(326.46345 .53)\end{array}$ & $\begin{array}{c}332.18 \\
(323.16341 .21)\end{array}$ & $<0.001^{*}$ \\
\hline Whole grain (gr/day) & $\begin{array}{c}19.59 \\
(17.7821 .39)\end{array}$ & $\begin{array}{c}80.35 \\
(75.6485 .06)\end{array}$ & $\begin{array}{c}34.80 \\
(32.4437 .16)\end{array}$ & $\begin{array}{c}39.18 \\
(37.0941 .26)\end{array}$ & 0.053 \\
\hline Nuts \& legumes (gr/day) & $\begin{array}{c}56.07 \\
(53.2158 .93)\end{array}$ & $\begin{array}{c}44.76 \\
(42.0547 .47)\end{array}$ & $\begin{array}{c}52.37 \\
(50.2754 .48)\end{array}$ & $\begin{array}{c}50.72 \\
(48.7452 .70)\end{array}$ & 0.14 \\
\hline $\begin{array}{l}\text { Sugar-sweetened } \\
\text { beverage (gr/day) }\end{array}$ & $\begin{array}{c}78.52 \\
(73.3084 .73)\end{array}$ & $\begin{array}{c}81.06 \\
(74.2887 .85)\end{array}$ & $\begin{array}{c}86.52 \\
(81.0791 .97)\end{array}$ & $\begin{array}{c}76.51 \\
(72.0580 .97)\end{array}$ & 0.97 \\
\hline Processed meat (gr/day) & $\begin{array}{c}24.05 \\
(22.4025 .70)\end{array}$ & $\begin{array}{c}30.18 \\
(28.1032 .26)\end{array}$ & $\begin{array}{c}35.06 \\
(33.2236 .90)\end{array}$ & $\begin{array}{c}38.36 \\
(36.9540 .13)\end{array}$ & $<0.001^{*}$ \\
\hline $\begin{array}{l}\text { Healthy eating index } \\
\text { (HEI) }\end{array}$ & $\begin{array}{c}47.19 \\
(46.5447 .85)\end{array}$ & $\begin{array}{c}48.88 \\
(48.3149 .45)\end{array}$ & $\begin{array}{c}48.72 \\
(48.2949 .15)\end{array}$ & $\begin{array}{c}49.62 \\
(49.0150 .23)\end{array}$ & $<0.001^{*}$ \\
\hline
\end{tabular}

*p-value $<0.05$ considered as statistically significant

inverse association was observed between age group and diet quality scores which is consistent with earlier studies. ${ }^{3,13,14}$ In addition, the greatest disparity in diet quality in relation to socio-demographic variables was for SES of living area, followed by family SES, gender and 
Table IV. Body composition and dietary intakes of children according to age and gender.

\begin{tabular}{|c|c|c|c|c|c|c|}
\hline \multirow{2}{*}{ Mean $(95 \% \mathrm{CI})$} & \multicolumn{2}{|c|}{ Gender } & \multirow{2}{*}{ P-value } & \multicolumn{2}{|c|}{ Age } & \multirow{2}{*}{ P-value } \\
\hline & Girl & Boy & & 6-12 years & 13-18 years & \\
\hline Body weight (kg) & $\begin{array}{c}42.18 \\
(41.4742 .88)\end{array}$ & $\begin{array}{c}42.04 \\
(41.3142 .78)\end{array}$ & 0.08 & $\begin{array}{c}33.44 \\
(3333.88)\end{array}$ & $\begin{array}{c}56.05 \\
(55.3156 .70)\end{array}$ & $<0.001^{*}$ \\
\hline $\begin{array}{l}\text { Waist } \\
\text { circumference }(\mathrm{cm})\end{array}$ & $\begin{array}{c}65.81 \\
(65.2866 .33)\end{array}$ & $\begin{array}{c}67.19 \\
(66.6867 .71)\end{array}$ & $0.015^{*}$ & $\begin{array}{c}62.23 \\
(61.8562 .63)\end{array}$ & $\begin{array}{c}73.40 \\
(72.8473 .93)\end{array}$ & $<0.001^{*}$ \\
\hline $\begin{array}{l}\text { Body mass index } \\
\left(\mathrm{kg} / \mathrm{m}^{2}\right)\end{array}$ & $\begin{array}{c}19.28 \\
(19.0619 .50)\end{array}$ & $\begin{array}{c}18.59 \\
(18.4118 .78)\end{array}$ & $<0.001^{*}$ & $\begin{array}{c}17.60 \\
(17.4417 .77)\end{array}$ & $\begin{array}{c}21.03 \\
(20.8121 .25)\end{array}$ & $<0.001^{*}$ \\
\hline $\begin{array}{l}\text { Total Energy } \\
\text { intake (Kcal/day) }\end{array}$ & $\begin{array}{c}2525.1 \\
(2492.82556 .4)\end{array}$ & $\begin{array}{c}2519.3 \\
(2483.82550 .7)\end{array}$ & 0.73 & $\begin{array}{c}2519.34 \\
(2489.92549 .6)\end{array}$ & $\begin{array}{c}25316 \\
(2493.72569 .2)\end{array}$ & 0.79 \\
\hline $\begin{array}{l}\text { Carbohydrate (\% } \\
\text { Kcal) }\end{array}$ & $\begin{array}{c}345.37 \\
(340.32350 .09)\end{array}$ & $\begin{array}{c}315.29 \\
(346.18355 .78)\end{array}$ & 0.12 & $\begin{array}{c}346.2 \\
(341.8350 .8)\end{array}$ & $\begin{array}{c}353.10 \\
(347.15358 .87)\end{array}$ & 0.07 \\
\hline Fat (\% Kcal) & $\begin{array}{c}96.22 \\
(94.6797 .78)\end{array}$ & $\begin{array}{c}92.97 \\
(91.3794 .48)\end{array}$ & $0.004^{*}$ & $\begin{array}{c}83.97 \\
(82.8885 .13)\end{array}$ & $\begin{array}{c}83.70 \\
(82.1985 .32)\end{array}$ & 0.12 \\
\hline Protein (\% Kcal) & $\begin{array}{c}84.09 \\
(82.8385 .34)\end{array}$ & $\begin{array}{c}83.62 \\
(82.3084 .90)\end{array}$ & 0.61 & $\begin{array}{c}95.11 \\
(93.7096 .52)\end{array}$ & $\begin{array}{c}93.75 \\
(91.9795 .45)\end{array}$ & 0.20 \\
\hline Fiber (gr/day) & $\begin{array}{c}27.81 \\
(27.2728 .34)\end{array}$ & $\begin{array}{c}28.70 \\
(28.1629 .26)\end{array}$ & 0.17 & $\begin{array}{c}27.96 \\
(27.4928 .44)\end{array}$ & $\begin{array}{c}28.77 \\
(28.1429 .42)\end{array}$ & 0.13 \\
\hline Sodium (mg/day) & $\begin{array}{c}6491.9 \\
(6049.17048 .1)\end{array}$ & $\begin{array}{c}5707.4 \\
(5411.86110 .7)\end{array}$ & $<0.001^{*}$ & $\begin{array}{c}5649.41 \\
(5308.26033 .6)\end{array}$ & $\begin{array}{c}6760.1 \\
(6320.017438 .8)\end{array}$ & $<0.001^{*}$ \\
\hline $\begin{array}{l}\text { Long chain omega } \\
3 \text { fatty acid }\end{array}$ & $\begin{array}{c}0.21 \\
(0.190 .23)\end{array}$ & $\begin{array}{c}0.22 \\
(0.200 .24)\end{array}$ & 0.45 & $\begin{array}{c}021 \\
(0.190 .23)\end{array}$ & $\begin{array}{c}0.22 \\
(0.200 .24)\end{array}$ & 0.26 \\
\hline $\begin{array}{l}\text { Poly unsaturated } \\
\text { fatty acid (gr/day) }\end{array}$ & $\begin{array}{c}18.24 \\
(17.8518 .63)\end{array}$ & $\begin{array}{c}17.76 \\
(17.4318 .09)\end{array}$ & 0.120 & $\begin{array}{c}17.90 \\
(17.59 \text { 18.22) }\end{array}$ & $\begin{array}{c}18.22 \\
(17.7918 .62)\end{array}$ & 0.56 \\
\hline Fruits (gr/day) & $\begin{array}{c}311.40 \\
(298.61324 .43)\end{array}$ & $\begin{array}{c}301.72 \\
(290.74314 .3)\end{array}$ & 0.08 & $\begin{array}{c}303.89 \\
(293.80314 .84)\end{array}$ & $\begin{array}{c}309.62 \\
(295.32323 .85)\end{array}$ & 0.18 \\
\hline $\begin{array}{l}\text { Vegetables (gr/ } \\
\text { day) }\end{array}$ & $\begin{array}{c}339.32 \\
(331.52347 .46)\end{array}$ & $\begin{array}{c}304.94 \\
(296.98312 .86)\end{array}$ & $<0.001^{*}$ & $\begin{array}{c}317.33 \\
(310.03325 .11)\end{array}$ & $\begin{array}{c}326.85 \\
(317.78336 .90)\end{array}$ & 0.07 \\
\hline $\begin{array}{l}\text { Whole grain (gr/ } \\
\text { day) }\end{array}$ & $\begin{array}{c}40.25 \\
(38.1342 .50)\end{array}$ & $\begin{array}{c}44.26 \\
(41.8446 .87)\end{array}$ & 0.55 & $\begin{array}{c}38.11 \\
(36.2140 .22)\end{array}$ & $\begin{array}{c}49.22 \\
(46.3852 .46)\end{array}$ & $<0.001^{*}$ \\
\hline $\begin{array}{l}\text { Nuts \& legumes } \\
\text { (gr/day) }\end{array}$ & $\begin{array}{c}48.80 \\
(47.0550 .78)\end{array}$ & $\begin{array}{c}51.76 \\
(49.8453 .58)\end{array}$ & $<0.001^{*}$ & $\begin{array}{c}50.25 \\
(48.7851 .85)\end{array}$ & $\begin{array}{c}50.21 \\
(48.1252 .35)\end{array}$ & $0.038^{*}$ \\
\hline $\begin{array}{l}\text { Sugar-sweetened } \\
\text { beverage (gr/day) }\end{array}$ & $\begin{array}{c}79.64 \\
(74.7884 .36)\end{array}$ & $\begin{array}{c}86.80 \\
(82.8891 .03)\end{array}$ & $<0.001^{*}$ & $\begin{array}{c}82.48 \\
(87.4786 .53)\end{array}$ & $\begin{array}{c}85.23 \\
(80.1490 .39)\end{array}$ & $0.008^{*}$ \\
\hline $\begin{array}{l}\text { Processed meat } \\
\text { (gr/day) }\end{array}$ & $\begin{array}{c}32.72 \\
(31.1834 .27)\end{array}$ & $\begin{array}{c}32.37 \\
(30.8433 .82)\end{array}$ & 0.48 & $\begin{array}{c}31.36 \\
(30.1232 .62)\end{array}$ & $\begin{array}{c}34.52 \\
(32.6436 .30)\end{array}$ & 0.12 \\
\hline $\begin{array}{l}\text { Healthy eating } \\
\text { index (HEI) }\end{array}$ & $\begin{array}{c}48.39 \\
(47.9748 .79)\end{array}$ & $\begin{array}{c}47.51 \\
(47.1447 .88)\end{array}$ & $<0.001^{*}$ & $\begin{array}{c}48.25 \\
(47.9148 .58)\end{array}$ & $\begin{array}{c}47.40 \\
(46.9647 .82)\end{array}$ & $0.003^{*}$ \\
\hline
\end{tabular}

${ }^{*}$ p-value $<0.05$ considered as statistically significant.

age. Results of the present study showed that people who are living in the south-east area had poorer diet quality due to lower intake of protein, fiber, fruits, vegetable and higher intake of fats. These results are in line with earlier studies that show a significant link between the socioeconomic status of the living region with diet quality in a way that those living in 
Table V. Association between socio-demographic variables and HEI by multilevel ordinal logistic regression.

\begin{tabular}{|c|c|c|c|c|c|c|c|c|}
\hline \multirow{2}{*}{\multicolumn{3}{|c|}{ Socio-demographic variables }} & \multicolumn{4}{|c|}{ HEI (percent) } & \multirow{2}{*}{$\begin{array}{c}\text { OR } \\
(95 \% \mathrm{CI})\end{array}$} & \multirow{2}{*}{ P-value } \\
\hline & & & Q1 & Q2 & Q3 & $\mathrm{Q} 4$ & & \\
\hline \multirow{7}{*}{$\begin{array}{l}\text { Individual- } \\
\text { level }\end{array}$} & \multirow[b]{2}{*}{ Age } & 6-12 years & $58.4 \%$ & $62.2 \%$ & $63.2 \%$ & $62.8 \%$ & 1 & \multirow[b]{2}{*}{$0.001^{*}$} \\
\hline & & $13-18$ years & $41.6 \%$ & $37.4 \%$ & $36.8 \%$ & $37.2 \%$ & $\begin{array}{c}0.76 \\
(0.640 .89)\end{array}$ & \\
\hline & \multirow[b]{2}{*}{ Sex } & Boy & $57.3 \%$ & $52.4 \%$ & $50.3 \%$ & $50.3 \%$ & 1 & \multirow[b]{2}{*}{0.07} \\
\hline & & Girl & $42.7 \%$ & $47.6 \%$ & $49.7 \%$ & $49.7 \%$ & $\begin{array}{c}1.15 \\
(0.991 .32)\end{array}$ & \\
\hline & \multirow{3}{*}{$\begin{array}{l}\text { Family socio- } \\
\text { economic status }\end{array}$} & Low & $37.8 \%$ & $34.9 \%$ & $34.4 \%$ & $26 \%$ & 1 & \\
\hline & & Moderate & $31.3 \%$ & $32.4 \%$ & $34 \%$ & $35.6 \%$ & $\begin{array}{c}1.30 \\
(1.131 .49)\end{array}$ & $<0.001^{*}$ \\
\hline & & High & $30.8 \%$ & $32.7 \%$ & $31.6 \%$ & $38.2 \%$ & $\begin{array}{c}1.36 \\
(1.181 .57)\end{array}$ & $<0.001^{*}$ \\
\hline \multirow{4}{*}{$\begin{array}{l}\text { Provincial- } \\
\text { level }\end{array}$} & \multirow{4}{*}{$\begin{array}{l}\text { Socio-economic } \\
\text { status of living } \\
\text { region }\end{array}$} & Lowest (Southeast) & $19.4 \%$ & $17.5 \%$ & $13 \%$ & $13.8 \%$ & 1 & \\
\hline & & $\begin{array}{l}\text { Second Low (North/ } \\
\text { Northeast) }\end{array}$ & $20.2 \%$ & $17.2 \%$ & $19.2 \%$ & $21.5 \%$ & $\begin{array}{c}1.19 \\
(0.532 .65)\end{array}$ & 0.68 \\
\hline & & $\begin{array}{l}\text { Second High SES } \\
\text { (West) }\end{array}$ & $31.1 \%$ & $30.1 \%$ & $31.5 \%$ & $29.6 \%$ & $\begin{array}{c}0.90 \\
(0.451 .80)\end{array}$ & 0.76 \\
\hline & & $\begin{array}{l}\text { Highest SES } \\
\text { (Central) }\end{array}$ & $29.3 \%$ & $35.2 \%$ & $36.3 \%$ & $35.1 \%$ & $\begin{array}{c}0.94 \\
(0.442 .02)\end{array}$ & 0.88 \\
\hline \multicolumn{3}{|c|}{ provincial level : Variance $(95 \% \mathrm{CI})$} & \multicolumn{6}{|c|}{$0.26(0.130 .53)$} \\
\hline
\end{tabular}

${ }^{*}$ p-value $<0.05$ considered as statistically significant.

HEI: healthy eating index.
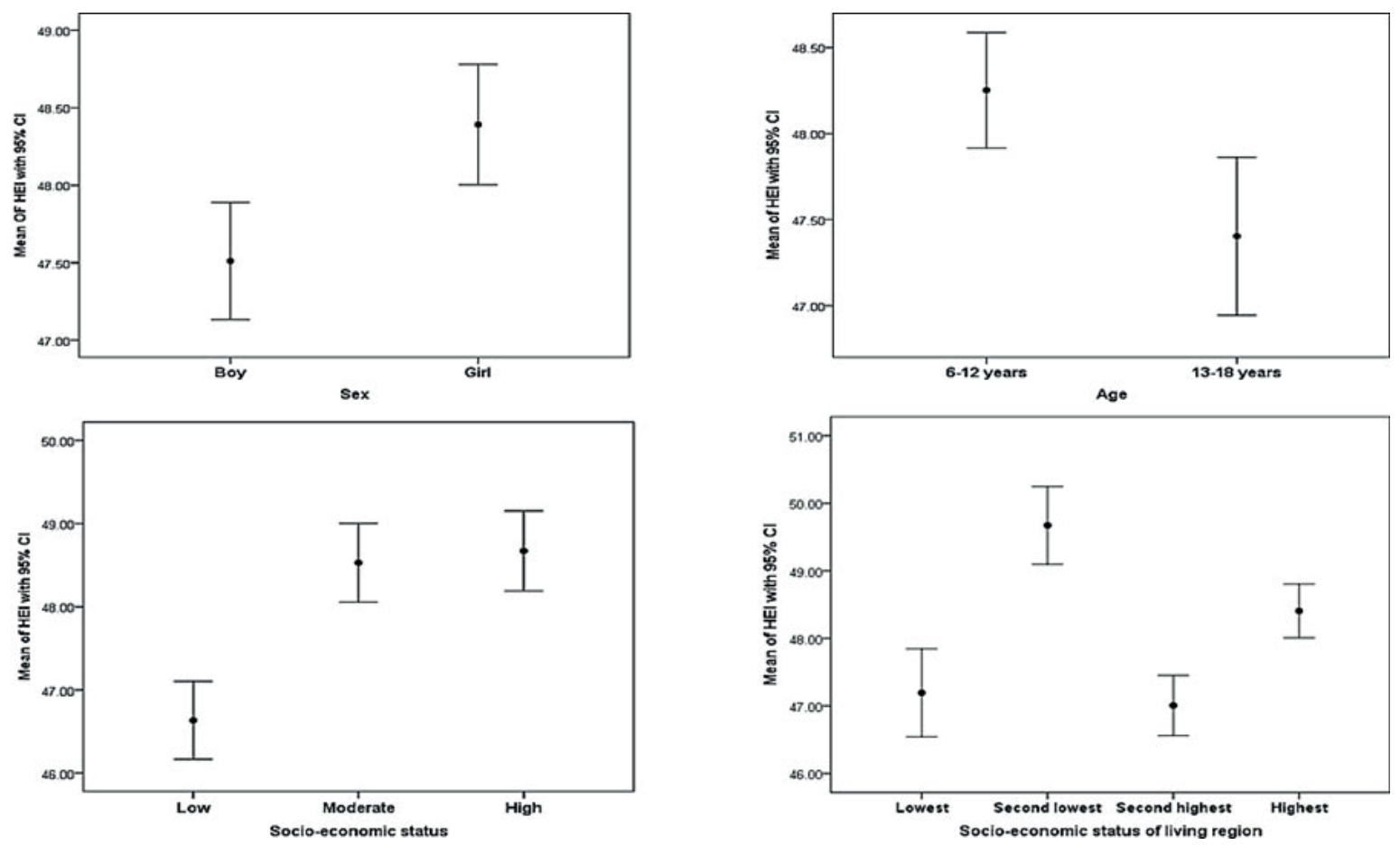

Fig. 1. HEI total mean scores and 95\% CI by socio-demographic characteristics. 
higher socioeconomic areas were more likely to have higher diet quality scores. ${ }^{4,7}$ One of the SES factors which according to the literature plays a barrier role for having a healthier diet is the level of family income. ${ }^{4,15}$ Recent metaanalysis and observational studies reported that healthier diet is more expensive than unhealthy ones. ${ }^{4,16-20}$ Analysis of the present study showed that children and adolescents in families with high SES had higher body weight and BMI with better diet quality than those in the lower SES which is in line with earlier studies. ${ }^{4,7}$ Better diet quality among these subjects was due to a higher intake of protein, fiber, fruits and vegetables and a lower intake of fat. The possible explanation for such an association is related to education and knowledge, two components of socioeconomic status. ${ }^{22,23}$ In other word, studies justified that those in higher SES families had higher income, education and better knowledge about foods. ${ }^{24-26}$

In the present analysis, girls showed better diet quality than boys, a finding consistent with other researches. ${ }^{15,27,28}$ Better diet quality among girls despite a higher intake of sodium and fats was owing to a higher score for vegetables and a lower score for SSB. Earlier investigations suggest that women are more likely to select healthier foods to maintain their body weight. A healthy diet is considered a feminine pursuit by men ${ }^{29}$ that is why studies targeting men get better results in terms of healthy diet patterns. ${ }^{30}$ The smallest disparities in diet quality of the analysis's socio-demographic variables were for age. Adolescents aged 13-18 years old compared to children aged 6-12years old showed higher body weight, BMI and lower diet quality due to consuming more sodium, and SSB and fewer amounts of nuts and legumes. Studies show that adolescents make poorer food choices than other age groups containing a higher amount of fat, sugar and processed foods..$^{22,31}$ In fact, the greatest concern is for adolescents who have the worst diet quality and tend to further decline in healthy eating patterns. ${ }^{32}$ The strength of the present study that should be taken into account is a large nationally representative sample of
Iranian children and adolescents and the use of FFQ to assess dietary intakes. The limitation of the study is a cross-sectional nature that does not allow establishing a causal relationship.

To sum up, the overall diet quality of Iranian children and adolescents were poor compared to the maximum score of HEI. Moreover, socio-demographic variables notably age and family income classes play a role in the quality of eating. It seems that Iranian girls aged 6-12 years old in families with high SES and living in the central area had better dietary patterns.

\section{REFERENCES}

1. Agardh E, Allebeck P, Hallqvist J, Moradi T, Sidorchuk A. Type 2 diabetes incidence and socioeconomic position: a systematic review and metaanalysis. IntjJ Epidemiol 2011; 40: 804-818.

2. McLaren L. Socioeconomic status and obesity. Epidemiol rRev 2007; 29: 29-48.

3. Thomson JL, Tussing-Humphreys LM, Goodman $\mathrm{MH}$, Landry AS. Diet quality in a nationally representative sample of American children by sociodemographic characteristics. Am J Clin Nutr 2019; 109: 127-138.

4. Grech A, Sui Z, Siu HY, Zheng M, Allman-Farinelli M, Rangan A. Socio-demographic determinants of diet quality in Australian adults using the validated Healthy Eating Index for Australian Adults (HEIFA-2013). Healthcare (Basel)2017; 5: 7.

5. Wirt A, Collins CE. Diet quality-what is it and does it matter? Public Health Nutr 2009; 12: 2473-2492.

6. Reedy J, Krebs-Smith SM, Miller PE, et al. Higher diet quality is associated with decreased risk of allcause, cardiovascular disease, and cancer mortality among older adults. J Nutr 2014; 144: 881-889.

7. Backholer K, Spencer E, Gearon E, et al. The association between socio-economic position and diet quality in Australian adults. Public Health Nutr 2016; 19: 477-485.

8. de Andrade SC, de Azevedo Barros MB, Carandina L, Goldbaum M, Cesar CLG, Fisberg RM. Dietary quality index and associated factors among adolescents of the state of Sao Paulo, Brazil. J Pediatr 2010; 156: 456-460.

9. Rafiei M, Boshtam M, Marandi A, Jalali A, Vakili R. The Iranian food consumption program (IFCP), a unique nutritional software in Iran. Iranian J Publ Health 2002: 31: 105-107. 
10. Dorosti Motlagh AR, M. T. Iranain Food Composition Table. (1 ed). . Iran: Donyaye Taghzieh Press, 2007.

11. Safiri S, Kelishadi R, Heshmat R, et al. Socioeconomic inequality in oral health behavior in Iranian children and adolescents by the Oaxaca-Blinder decomposition method: the CASPIAN-IV study. Int J Equity Health 2016; 15: 143.

12. Täger $M$, Peltner J, Thiele S. Evaluation of diet quality by means of the Healthy Eating Index and its modified variants. Ernährungs Umschau 2016; 63: 110-118.

13. Banfield EC, Liu Y, Davis JS, Chang S, Frazier-Wood AC. Poor adherence to US dietary guidelines for children and adolescents in the national health and nutrition examination survey population. J Acad Nutr Diet 2016; 116: 21-27.

14. Gu X, Tucker KL. Dietary quality of the US child and adolescent population: trends from 1999 to 2012 and associations with the use of federal nutrition assistance programs. Am J Clin Nutr 2016; 105: 194202.

15. Hiza HA, Casavale KO, Guenther PM, Davis CA. Diet quality of Americans differs by age, sex, race/ ethnicity, income, and education level. J Acad Nutr Diet 2013; 113: 297-306.

16. Drewnowski A, Darmon N. Food choices and diet costs: an economic analysis. J Nutr 2005; 135: 900904.

17. Maillot M, Darmon N, Vieux F, Drewnowski A. Low energy density and high nutritional quality are each associated with higher diet costs in French adults. Am J Clin Nutr 2007; 86: 690-696.

18. Rao M, Afshin A, Singh G, Mozaffarian D. Do healthier foods and diet patterns cost more than less healthy options? A systematic review and metaanalysis. BMJ Open 2013; 3: e004277.

19. Afshin A, Peñalvo JL, Del Gobbo L, et al. The prospective impact of food pricing on improving dietary consumption: a systematic review and metaanalysis. PloS One 2017; 12: e0172277.

20. Kern D, Auchincloss AH, Stehr MF, et al. Neighborhood prices of healthier and unhealthier foods and associations with diet quality: evidence from the multi-ethnic study of atherosclerosis. Int J Environ Res Public Health2017; 14: 1394.

21. Arabshahi S, Lahmann PH, Williams GM, Marks GC, van der Pols JC. Longitudinal change in diet quality in Australian adults varies by demographic, socio-economic, and lifestyle characteristics. J Nutr 2011; 141: 1871-1879.
22. Turrell G, Kavanagh AM. Socio-economic pathways to diet: modelling the association between socioeconomic position and food purchasing behaviour. Public Health Nutr 2006; 9: 375-383.

23. Beydoun MA, Wang Y. How do socio-economic status, perceived economic barriers and nutritional benefits affect quality of dietary intake among US adults? Eur J Clin Nutr 2008; 62: 303-313.

24. Assumpção D, Domene SMÁ, Fisberg RM, Barros MBA. Social and demographic inequalities in diet quality in a population-based study. Rev Nutr 2016; 29: 151-162.

25. Morimoto JM, Latorre MdRDd, César CLG, Carandina L, Barros MBdA, Goldbaum M, et al. Fatores associados à qualidade da dieta de adultos residentes na Região Metropolitana de São Paulo, Brasil, 2002. CadSaúde Pública 2008; 24: 169-178.

26. Freitas TI, Previdelli AN, Ferreira MPN, Marques KM, Goulart RMM, Aquino RC. Factors associated with diet quality of older adults. Rev Nutr 2017; 30: 297-306.

27. van Lee L, Geelen A, Kiefte-de Jong JC, et al. Adherence to the Dutch dietary guidelines is inversely associated with 20-year mortality in a large prospective cohort study. Eur J Clin Nutr 2016; 70: 262-268.

28. McNaughton SA, Ball K, Crawford D, Mishra GD. An index of diet and eating patterns is a valid measure of diet quality in an Australian population. TJ Nutr 2008; 138: 86-93.

29. Robertson C, Avenell A, Boachie C, et al. Should weight loss and maintenance programmes be designed differently for men? A systematic review of long-term randomised controlled trials presenting data for men and women: The ROMEO project. Obes Res Clin Pract 2016; 10: 70-84.

30. Hunt K, Wyke S, Gray CM, et al. A gender-sensitised weight loss and healthy living programme for overweight and obese men delivered by Scottish Premier League football clubs (FFIT): a pragmatic randomised controlled trial. Lancet 2014; 383: 12111221.

31. van Lee L, Geelen A, Kiefte-de Jong JC, et al. Adherence to the Dutch dietary guidelines is inversely associated with 20-year mortality in a large prospective cohort study. Eur J Clin Nutr 2016; 70: 262-628.

32. Andrade SC, Previdelli AN, Cesar CLG, Marchioni DM, Fisberg RM. Trends in diet quality among adolescents, adults and older adults: a populationbased study. Prev Med Rep 2016; 4: 391-396. 